\title{
Are Prominences Formed by Flux Convergence?
}

\author{
K. Galsgaard and A. W. Longbottom
}

Department of Mathematical and Computational Sciences, University of St. Andrews, St. Andrews, North Haugh, KY16 9SS, Scotland

\begin{abstract}
Numerical 3D resistive MHD experiments are used to investigate the dynamical properties of a quadrupolar magnetic field, as the two dipolar regions are pushed together. A current concentration is formed between the approaching flux concentrations and, when reconnection starts, material is lifted against gravity. As it rises the material spreads out along the polarity inversion line and forms a prominence-like structure.
\end{abstract}

\section{Introduction}

Quiescent prominences have been observed intensely for decades. Despite much effort into understanding them they are still mysterious phenomena, providing many unanswered questions. One such question relates to their method of formation. At present there are no comprehensive observations that provide detailed information about how prominences form. However, indirect quantities have been extracted from the huge observational material. Martin (1990) analysed regions where prominences had formed, prior to their formation, and found three necessary criteria for prominence formation. These are: 1) the presence of a magnetic arcade overlying a polarity inversion line; 2) the convergence of flux towards the polarity inversion line under the arcade; 3 ) the cancellation of opposite flux in the vicinity of the polarity inversion line. The first two points provide clear information about topology and flow dynamics, but the third point, the cancellation of flux, is by no means clear. The observations have not been able to distinguish between flux emergence/submergence of bipolar regions close to the polarity inversion line, and annihilation of magnetic flux due to magnetic reconnection. The difference is important and will, when resolved, put strong limitations on models describing this important phase of prominences life.

To put forward a simple model for prominence formation, a quadrupolar magnetic field is taken as the initial magnetic field configuration. This magnetic field topology contains the first of Martin's (1990) criteria, and has a simplified topology similar to the one example discussed by MacKay et al. (1997). The topology of the magnetic field is shown in Figure 1. The sources contain the same amount of flux, though their surface areas are different. The magnetic field is divided into four topologically distinct regions by the separator surfaces defined by two 3D null points located at the photospheric boundary. The magnetic field in the Cartesian box is found by solving for the potential solution to the imposed boundary conditions (Longbottom et al. 1997), assuming that magnetic flux is only penetrating the bottom boundary. As magnetic field is only penetrating 


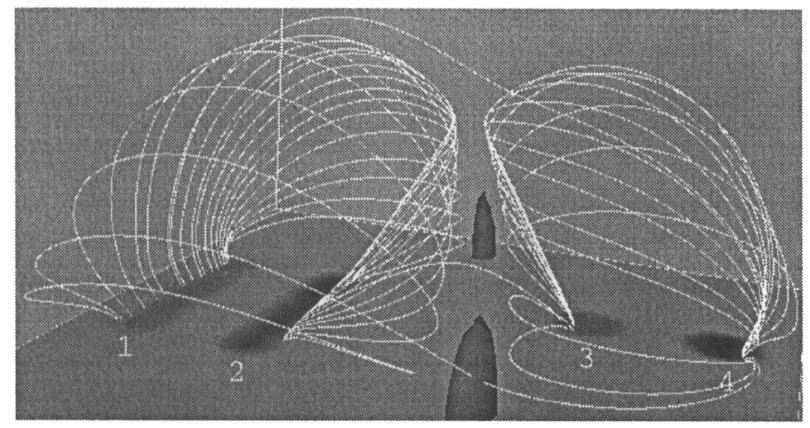

Figure 1. The initial magnetic field line topology. The shading at the bottom plane indicates the locations of the penetrating flux. Field line traces outline the location of the separator connecting the two nulls. The null point locations and the start of the separator line, that divides space into four magnetically independent flux regions, is indicated by the isosurfaces.

the "photospheric" boundary at the four source locations and then immediately becomes space filling, the magnetic field strength changes dramatically over a short distance close to the sources. To get time-scales in different parts of the numerical domain that are not too different, a model atmosphere with a density stratification with up to 12 scale heights and a constant plasma temperature is used.

To fulfill the second criteria of Martin (1990) a smooth step function velocity profile is imposed on the lower boundary. This moves the two sets of dipolar regions (1-2, 3-4 in Figure 1) toward each other without distorting their individual separation. The dynamical evolution is followed numerically by solving the full set of MHD equations (Nordlund and Galsgaard 1995, URL: http://wwwsolar.dcs.st-and.ac.uk/ ${ }^{\sim}$ klaus) without a proper treatment of heat conduction and plasma condensation.

\section{Results}

Experiments with different parameter values have been conducted, representing different magnetic field strengths and number of density scale heights. This paper only discusses the general features of the experiments, without giving details of the dependence on specific parameters (see Galsgaard and Longbottom 1998).

As the magnetic flux distributions are forced together by the boundary motion, the magnetic field between the two approaching flux concentrations is compressed. Initially, the connections from the strong circular flux distribution (3) connect to a thin line along the straight edge of the elongated flux distribution (2) with almost straight field lines when projected onto the bottom boundary. When the distance between sources 2 and 3 decreases, the field lines connecting them change shape. Close to the sources this change is minor while the weaker parts higher up in the atmosphere accumulate nearly all of the horizontal change 
a
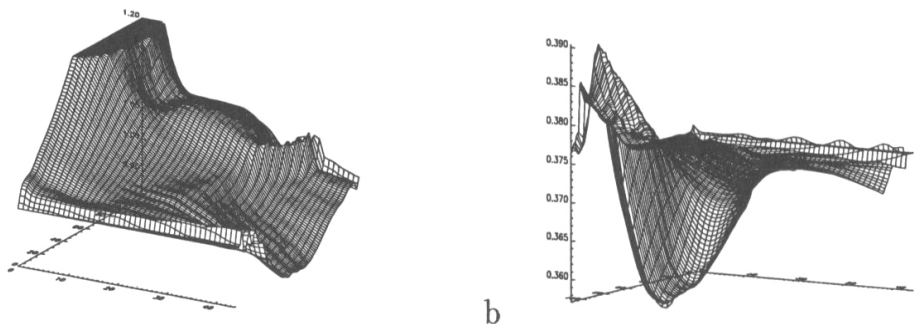

Figure 2. Left, the change in density (relative to the initial density) as a function of height and time measured on a line of data points located at the convergence line between the two approaching sources. Right, a similar plot for the plasma temperature.

along the convergence line between the footpoints and, therefore, become almost parallel to this line.

As the field between the two sources $(2,3)$ is compressed, magnetic field with different horizontal components approach each other and a current concentration is slowly built up between them. As the current magnitude increases, it eventually reaches a strength where the resistive and advective contributions to the induction equation become comparable, and the field line connectivity starts to change in the current sheet region. Half of these reconnected field lines have an upward directed tension force that is strong enough to lift dense plasma against gravity. As the field lines move away form the reconnection site, and the field line dip broadens, the captured dense plasma spreads out along the field line above the polarity inversion line. This decreases the absolute density, but maintains a density enhancement relative the surrounding plasma, a possible first step in a prominence formation.

Figure 2a shows the change in density relative to the initial distribution as a function of height and time for a line of points between the two approaching flux sources that are located on the center of the convergence line. Here it is seen that the density close to the base grows almost linearly with time until about $t=15$, when the reconnection starts. The buildup of density in the initial phase is due to the fact that the plasma is trapped on field lines that are slowly shortened and that the plasma is not allowed to exit the box through the boundaries. After $t=15$ a flat density plateau is formed above a certain height - the "prominence" location - while the density below increases rapidly because the field lines are now much shorter and are simultaneous being compressed by the dominating downward directed tension force. At the base the large density enhancement is not spread out in the horizontal direction, because the magnetic field is strong enough to confine it. Despite the Joule heating in the current sheet, it is found that the lifted plasma decreases in temperature as it is lifted. The material is lifted almost adiabatically and, therefore, has to adjust to the lower surrounding plasma pressure by decreasing it's plasma density (spreading out along the field lines) and decreasing the temperature. This mechanism forms a low temperature blob, Figure $2 \mathrm{~b}$, which for a long period of time is the easiest way of identifying the field lines that have changed connectivity. 


\section{Conclusion}

The experiments have shown that it is possible to lift plasma against gravity by magnetic field lines that reconnect close to the bottom boundary as a part of the process of flux convergence. They also show that the lift height and the density enhancement increases with decreasing magnetic $\beta$, a natural consequence of the increasing influence of the magnetic forces on the plasma dynamics. The lift velocity of the plasma is always found to peak around the Alfvenic Mach one just above the location of the peak tension force. One would expect to find similar lift velocities on the sun, if this process is important for any phase of a prominences' life. The experiments do not have realistic values of magnetic $\beta$, nor do they include a transition region which we expect is required to be located between the reconnection site - in the lower chromosphere - and the prominence - in the corona - to obtain a significant density enhancement relative to the plasma surrounding the prominence. It is found that the density enhancement starts to decrease as soon as the driving of the system is turned off. Therefore, to maintain the prominence for a long time, there has to be a continued flux convergence beneath it that can provide a continued mass input. When the mass supply stops, the density plasma slowly drains away from the prominence region. This occurs because practically none of the field lines passing through the prominence are spiralling along the prominence axis. The majority of field lines supporting dense plasma pass through the prominence region, having summit points on either side of the prominence before connecting back to the sources on the bottom boundary. It is found that the height difference between the summit and the dip supporting the dense plasma decreases with time. The plasma therefore slowly slips over the lowest of the summit points and drains down towards the bottom boundary.

Our expectation is, therefore, that to maintain a prominence, built by the principle of flux convergence for a long period of time, there must be a continued mass supply replacing the plasma that is slowly draining down. The life of a prominence must therefore be a highly dynamical phenomena that will only be fully understood when investigated under this assumption. To obtain such insight it is of vital importance that high resolution observations of plasma motions - velocities, masses - and magnetic fields are obtained. Only such observations can provide the information required to understand the life of prominences.

Acknowledgments. We acknowledge the support by PPARC, providing financial funding for our positions and computing time on the T3D at the Edinburgh Parallel Computer Center.

\section{References}

Galsgaard, K. and Longbottom, A. W. 1998, ApJ, in preparation Longbottom, A. W. et. al. 1997, A\&AS, submitted

Martin, S. F. 1990, in Dynamics of Quiescient Prominences, (eds.) V. Ruzdjah and E. Tandberg-Hansen, Springer-Verlag, New York, p. 1

MacKay, D. et al. 1997, ApJ, 486, 534 